\title{
EDITORIAL
}

\section{RESEARCH PRODUCTIVITY IN SRI LANKA}

Many senior scientists are agreed in their dismay at the apparent decline in the scientific competence of our nation. One of the indicators of productivity is the output of research papers. Sri Lanka does not publish a single journat that figures in the Science Citation Index. In comparison, among our neighbours India counts 48, Pakistan 2 and Bangladesh 2. Among local publications, the "Journal of the National Science Foundation", by its wide circulation and broad coverage of disciplines, is best placed to secure this recognition.

Conscious of this need, the Editorial Board has been actively engaged in securing the help of eminent and recognized scientists from abroad who represent high levels of achievement in a variety of disciplines and fields, to constitute an International Panel of referees. Local scientists already, by and large, respond graciously to our requests for referral help.

Other criteria to be met by a journal to merit inclusion in a Scientific Index include regularity of publication, serious refereeing and of course, a consistently high standard in content and presentation.The Editorial Committee has rigorously endeavoured to meet these standards.

Presently, very few papers by Sri Lankan scientists figure in Indexing or Abstracting journals. And here too, the majority are from Sri Lankan nationals working in laboratories abroad. As an example, only 234 papers by Sri Lankans enjoyed this distinction in 2004 . In contrast, India counted 21 397, Pakistan 933 and Bangladesh 436. Disparities in population alone do not justify this depressing performance.

It has to be fairly admitted that the major part of local research is State financed and the bulk of investment is into State institutions. Their mandates and priorities necessarily focus on issues of local interest and importance and thus of limited interest to an international readership. Neither Sate-resourcing nor output are anywhere near adequate. Financial resources are not all that matter. Even the most charitable evaluation would be hard put to argue that the return is adequate even for the meager investment. Admittedly, low investment yields poor returns. Poor returns in turn prompt low investment.

Several factors have contributed to the present state. Some are incontestable, others are contentious. Poor syllabi implemented by inadequately motivated and qualified teachers, outdated instructional techniques, undue emphasis on examinations constitute a cluster. Where demand for education greatly outstrips the capacity to deliver, examinations become more a means of elimination than of selection. Teaching leans heavily towards what can be examined rather than what is useful to know. When educational content is dictated by the convenience of examining, originality and creativity will inexorably give way to memory recall. Research capability necessarily suffers from poor basic scientific preparation.

Vacillating approaches to the medium of instruction have impacted adversely on quality. Restriction of access to English, the most widely used language of Science restricts students to second hand or even worse versions of knowledge. The perils are clear.

Academic promotion systems that can overlook quality for quantity are patently destructive. Reward systems that confine themselves to recognizing publications in International Journals alone are unhelpful for nourishing local journals with quality papers.

The above few, and several other causes conspire to create a disastrous pattern. One result is a paucity of quality papers to sustain and improve the JNSF. The Editorial Board, averse to compromising on quality, has been compelled to resort to the device of "combined issues". We fervently desire an early return to the quarterly frequency.

Expanding the practice of entertaining mainly original research papers, we now consider short notes on important findings, articles on topical issues, comments on published articles, scientific correspondence and reviews of scientific books and 
as in the past, invited review articles. Our readers, we trust will notice and welcome the change that is apparent in this issue. Guidance for contributors to each category is included in our March 2005 Issue (Volume 33 No. 1).
Your co-operation in upgrading the value and recognition of the Journal of the National Science Foundation of Sri Lanka is most earnestly solicited.

\section{U. Pethiyagoda}

Former Editor-in-Chief

10.03.2005 